\title{
The importance of moisture distribution for the growth and energetics of mid-latitude systems
}

\author{
V. Pavan ${ }^{1}$, N. Hall ${ }^{2}$, P. Valdes and M. Blackburn ${ }^{3}$ \\ ${ }^{1}$ CINECA, via Magnanelli 6/3, 40033 Casalecchio di Reno, Bologna, Italy \\ ${ }^{2}$ Department of Atmospheric and Oceanic Sciences, McGill University, Montreal, Canada \\ ${ }^{3}$ Department of Meteorology, University of Reading, UK
}

Received: 3 February 1998 / Revised: 22 June 1998 / Accepted: 30 June 1998

\begin{abstract}
A primitive equation model is used to study the sensitivity of baroclinic wave life cycles to the initial latitude-height distribution of humidity. Diabatic heating is parametrized only as a consequence of condensation in regions of large-scale ascent. Experiments are performed in which the initial relative humidity is a simple function of model level, and in some cases latitude bands are specified which are initially relatively dry. It is found that the presence of moisture can either increase or decrease the peak eddy kinetic energy of the developing wave, depending on the initial moisture distribution. A relative abundance of moisture at midlatitudes tends to weaken the wave, while a relative abundance at low latitudes tends to strengthen it. This sensitivity exists because competing processes are at work. These processes are described in terms of energy box diagnostics. The most realistic case lies on the cusp of this sensitivity. Further physical parametrizations are then added, including surface fluxes and upright moist convection. These have the effect of increasing wave amplitude, but the sensitivity to initial conditions of relative humidity remains. Finally, 'control' and 'doubled $\mathrm{CO}_{2}$ ' life cycles are performed, with initial conditions taken from the time-mean zonal-mean output of equilibrium GCM experiments. The attenuation of the wave resulting from reduced baroclinicity is more pronounced than any effect due to changes in initial moisture.
\end{abstract}

Key words. Meteorology and atmospheric dynamics (climatology; convective processes; synoptic-scale meteorology)

\section{Introduction}

Discussion of long-term trends in our climate has often been centred on uncertainties in the role of changing humidity (see e.g. Held, 1993; Shine and Sinha, 1991; Mitchell et al., 1989). This is not only true for the equilibrium, or slowly varying aspects of climate change, but also for the transient eddies. In mid-latitudes, weather systems of the order of $1000 \mathrm{~km}$ in scale dominate the transient fluxes of heat, momentum and water vapour. These fluxes form a major part of the forcing which maintains the climatological flow. The response of synoptic systems to changing climatic conditions is also of pivotal importance for local impacts. An understanding of the effect of changing moisture distributions on these systems could therefore be crucial to our understanding of climate change.

Mid-latitude transient activity depends in turn on prevailing climatic conditions through the average structures of temperature, wind and humidity. Simple modelling studies indicate that transient activity may respond to a change in average temperature structure in a relatively straightforward way. In a global warming scenario, for example, lower tropospheric temperature gradients are typically reduced. Idealized studies by (Branscome et al., 1992; Pavan, 1995) show an accompanying reduction in storminess, entirely owing to the dry dynamics of baroclinic waves. Diagnosis of GCM simulations of global warming (Boer, 1993; Hall et al., 1994) and the last ice age (Hall et al., 1996) often shows similar consistency. However, marked differences can exist between GCMs (Mitchell et al., 1990) in relation to the important variations of low-level temperature with both latitude and longitude. Furthermore, the presence of two distinct 'storm tracks' in the Northern Hemisphere, and the contribution of altered time-mean stationary waves to cross-latitude fluxes can complicate the picture considerably. 
The presence of moisture adds a further level of complication. Its various effects range from direct to subtle and can be of opposing sign. Numerous studies (Chang et al., 1982; Fantini, 1995) indicate that the growth rates of short baroclinic waves can be increased by condensation heating in phase with the temperature structure of the wave, creating a source of eddy available potential energy. To a lesser extent, this direct energy input to the wave can also be a factor for longer-wave, synoptic scale systems. On the other hand, condensation heating in relatively cold air could alter the baroclinicity of the flow in such a way as to deplete the zonal mean available potential energy, leading to a reduction in wave growth. This second mechanism would be consistent with the poleward transport of water vapour away from its source region before it condenses. Horizontal flux of latent heat would replace horizontal flux of sensible heat in the energy budget, allowing for less active transients. This could be important in a warmer, moister world (Boer, 1993). Finally, it is possible that the enhanced vertical motion associated with condensation heating could accelerate existing energy conversions, reinforcing wave growth.

These mechanisms are difficult to isolate in a GCM, so simple modelling studies remain our best guide. One approach is to study baroclinic wave life cycles, of the type pioneered by Simmons and Hoskins (1978). The problem of diagnosing mechanisms is simplified to a manageable level by working with a single eddy. The assumption is that this single eddy can represent the ensemble of eddies that we see in reality. Possible interactions between the eddy and changes in its environment as it propagates are ignored, except for those which arise directly from the alteration of the zonal flow by the eddy itself. Gutowski et al. (1992) have made extensive use of these types of calculations to diagnose the possible effects of moisture on mid-latitude systems. They find that the dominant response to condensation heating is thermally direct, with enhanced ascent in the warm air assisting the conversion of eddy available potential energy to eddy kinetic energy, resulting in a larger amplitude wave.

In the context of climate change, the same authors have taken the zonal mean climates from various GCM simulations with doubled $\mathrm{CO}_{2}$ and used them as initial conditions for life-cycle experiments. They find that the effect of reduced meridional temperature gradients usually (Branscome et al., 1992), but not always (Gutowski et al., 1995), outweighs the enhancement of the wave due to moisture. The results from the GCMs themselves show considerable variation in mean temperature gradients and humidity, both in their control simulations and in their predictions of climate change. There is, therefore, a need to examine further the sensitivity of mid-latitude systems to changes in moisture, in isolation from other effects and over a range of initial conditions.

This work shows results for several baroclinic wave life-cycle experiments with initial conditions which are, in part, taken from previous GCM experiments and, in part, idealised. We concentrate attention on the sensi- tivity of the wave growth to the initial moisture distribution. Because of the possibly competing effects already discussed, the locations at which condensation is likely to occur in the early stages of wave growth will probably be crucial to the life-cycle response. Therefore, the relevant variable whose initial distribution is to be examined is the relative humidity, since this is a measure of how close the air is to saturation. As the life-cycle progresses, water vapour will be transported by the developing wave, and the fact that warm saturated air contains more water vapour than cold saturated air will become important, but the initial distribution of relative humidity gives the best indication of where condensation is initially likely to occur. In the following experiments, the initial relative humidity is prescribed through simple functions of latitude and height. In this way we can identify sensitive regions, and compare these with the observed structure of relative humidity, and with known errors in GCMs. In Sect. 2, results are shown from experiments in which the only physical process we parametrize is the condensation of moisture present in the initial condition in ascending air. In diagnosing the results, we concentrate on the energetics of the systems as they develop. An interesting sensitivity emerges within a reasonable range of initial conditions. We argue that this sensitivity is of relevance when interpreting the results from more comprehensive climate simulations. Section 3 extends these results to include further physical parametrizations and in Sect. 4 we briefly describe a ' $2 \times \mathrm{CO}_{2}$ ' experiment using initial conditions from a GCM. Conclusions are given in Sect. 5.

\section{Large-scale precipitation}

\subsection{Experimental design}

All life-cycle experiments in this study are performed using the primitive equation model of Hoskins and Simmons (1975). Resolution is set at T42 with 11 sigma levels in the vertical corresponding to the levels in the UK Meteorological Office GCM (Mitchell et al., 1990). A six-fold symmetry is imposed around latitude circles, i.e. only zonal wave number six and its harmonics are included in the spectral series. The domain is also restricted to the Northern Hemisphere, with appropriate symmetries imposed at the equator. There is no orography and the only diabatic term apart from the parametrizations described below is $4 \mathrm{~h} \nabla^{6}$ hyper diffusion acting on vorticity, divergence and temperature.

Initial conditions have been taken from the equilibrium 'control' integration of the UK Meteorological Office GCM (Mitchell et al., 1990). Ten year mean, zonal means of temperature and surface pressure for the winter season (DJF) are input to a balancing procedure to deduce the zonal winds. These fields are shown in Fig. 1. They form a representative atmospheric state which is adequate for our purposes. life cycles are initiated by adding a small perturbation to the initial 


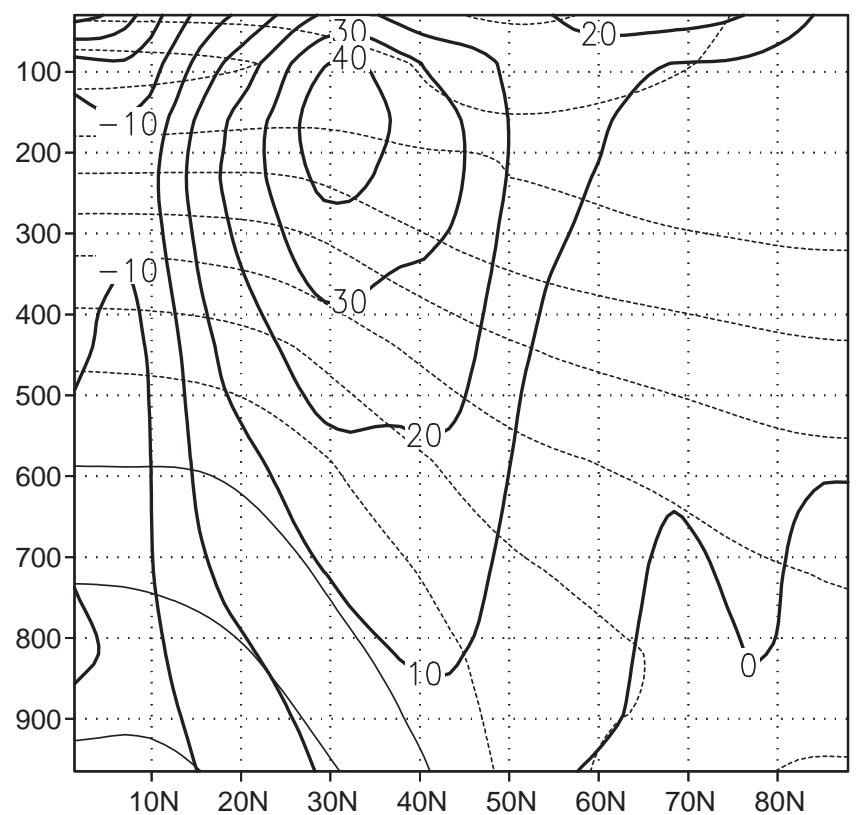

Fig. 1. Zonal mean base state taken from the control integration of the UK Meteorological Office climate model. Thick solid contours show zonal wind (contour interval $10 \mathrm{~m} \mathrm{~s}^{-1}$ ). Lighter contours show temperature (contour interval $10^{\circ} \mathrm{C}$, solid and zero contours solid, negative contours dashed)

condition with the structure of the fastest growing linear normal mode with zonal wave number 6 .

The observed zonal mean winter-time mean relative humidity (1979-1995, NCEP National Centre for Environmental Prediction reanalysis, see Kalnay et al., 1996) is shown in Fig. 2, expressed as a percentage. At a given latitude, it is almost a linear function of pressure, and so to a first approximation it can be represented in our model as a constant times the vertical coordinate, sigma.
This constant should lie somewhere between 60 and 100 . There is an obvious dip in relative humidity in subtropical regions where a value of 60 would be more appropriate. There are maxima at the equator and at mid-latitudes where the constant should be closer to 100 .

As an initial test of sensitivity, three experiments are shown with the constant independent of latitude and set to zero (DRY), 60 (G60), and 100 (G100). Two more experiments are then shown with the constant set to 100, but with a relatively dry region where the constant is 60 . One experiment has the relatively dry region in the subtropics, between $10^{\circ} \mathrm{N}$ and $35^{\circ} \mathrm{N}$ (G100ST60). The other has it in mid-latitudes between $35^{\circ} \mathrm{N}$ and $50^{\circ} \mathrm{N}$ (G100ML60).

The life-cycle experiments which have moisture in the initial condition include a simple parametrization of heating due to condensation. At each time step, at each grid point, if the air becomes super-saturated, an adjustment is made to the specific humidity $q$, and the temperature $T$, which satisfies the relation

$c_{p} \triangle T=-L \triangle q$,

such that the air is no longer super-saturated. The excess water vapour $\Delta q$, is immediately precipitated out of the model without evaporation. This parametrization, and those in the next section, are switched on at day three to allow the dry normal mode to develop to a reasonable amplitude before it is perturbed.

\subsection{Results}

The results shown in this section come from experiments where the only physical parametrization is that of heating due to condensation in areas of ascent. Figure 3a shows the development of eddy kinetic energy $\left(K_{e}\right)$ in the DRY, G100 and G60 life cycles described already.

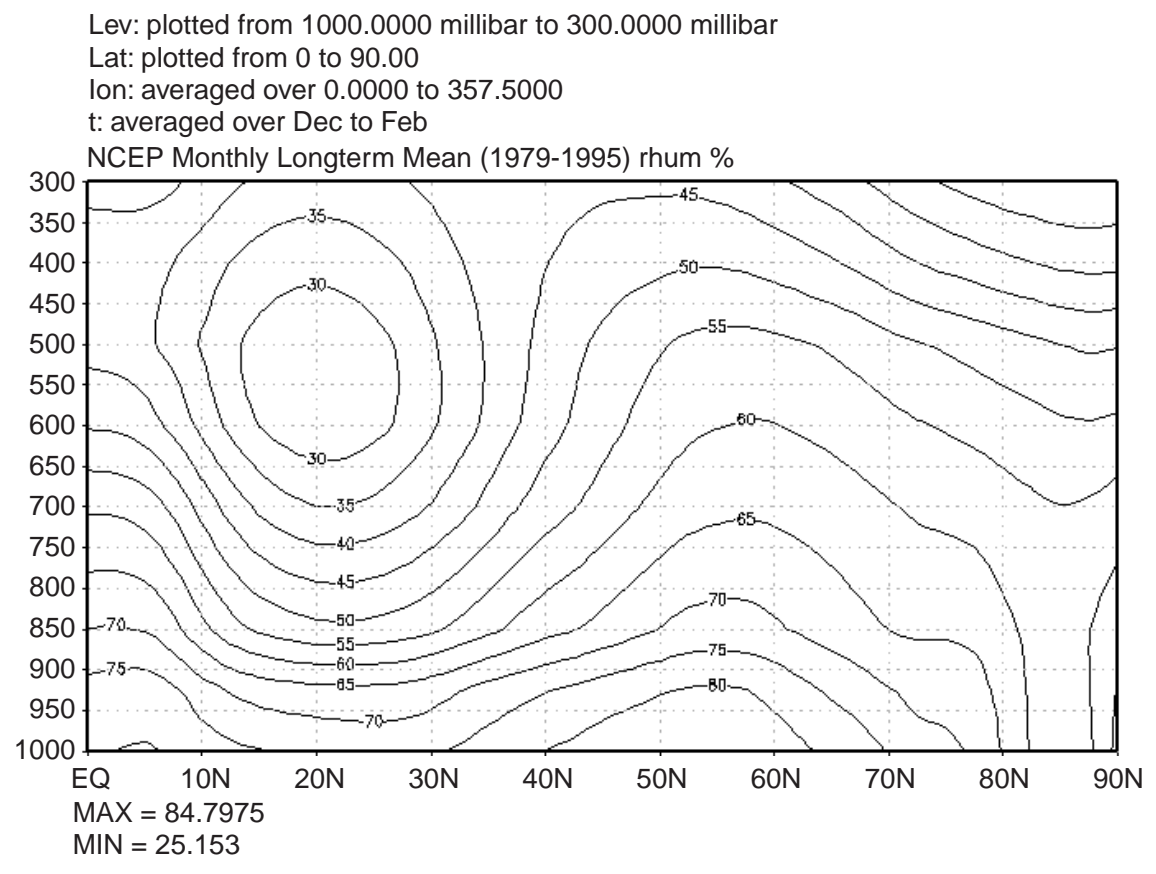

Fig. 2. 1979 to 1995 long-term mean of zonal mean wintertime (DJF) relative humidity as a function of latitude and pressure for the Northern Hemisphere (NCEP reanalysis data). Values given are percentages. contours every $5 \%$ 
a

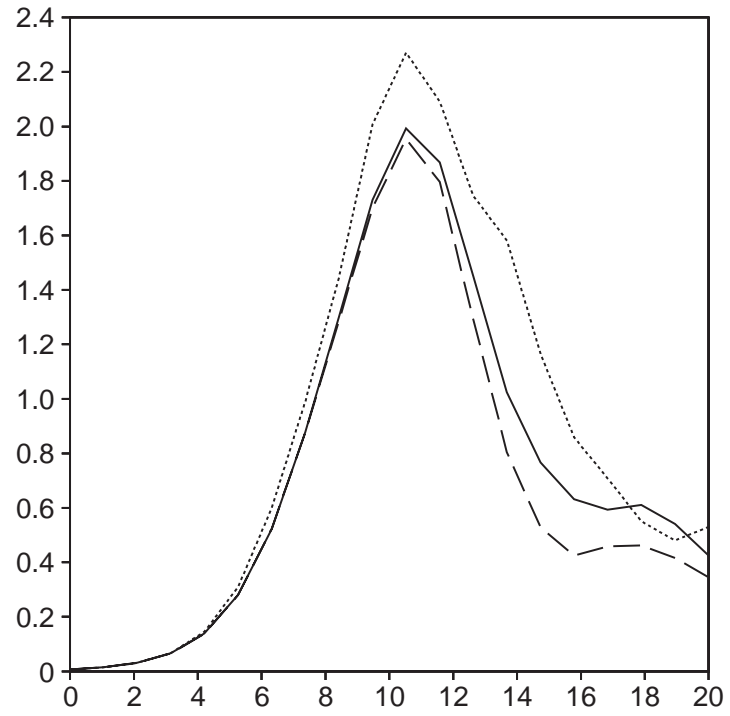

b

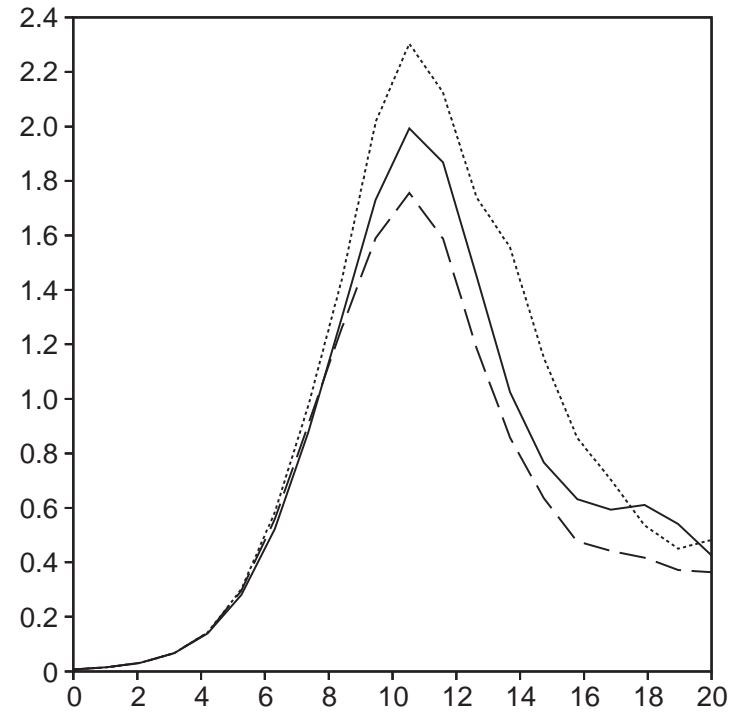

Fig. 3a,b. Time development of eddy kinetic energy, $K_{e}$ for five life cycle experiments. a DRY (solid curve), G60 (dashed) and G100 (dotted). b DRY (solid curve), G100ST60 (dashed) and G100ML60 (dotted). Values plotted in units of $10^{5} \mathrm{~J} \mathrm{~m}^{-2}$

All life cycles start with exponential growth, saturate at a maximum $K_{e}$ on day 11 and then decay. The DRY experiment has a maximum $K_{e}$ of $1.95 \times 10^{5} \mathrm{~J} \mathrm{~m}^{-2}$. The maximum $K_{e}$ is increased to $2.25 \times 10^{5} \mathrm{~J} \mathrm{~m}^{-2}$ in the G100 experiment, where the presence of condensation heating clearly enhances the growth of the wave. However, if a little less moisture is added to the initial condition, this enhancement disappears. A separate 'G90' experiment (not shown) confirms this. The G60 experiment actually has a maximum $K_{e}$ which is slightly reduced compared to the DRY experiment. In this case, the presence of condensation heating slightly attenuates the growth of the wave.

The results from the other two moist experiments outlined are shown in Fig. 3b, along with the DRY curve which is shown again for reference. These experiments have relative humidity which is equal to $100 \% \times \sigma$ everywhere except in a relatively dry latitude band where it is $60 \% \times \sigma$. The experiment with the drier subtropics (G100ST60) now shows a marked reduction in maximum $K_{e}$ compared to the DRY experiment. It is reduced to a value of $1.75 \times 10^{5} \mathrm{~J} \mathrm{~m}^{-2}$. In contrast, the experiment with the drier mid-latitudes (G100ML60) shows increased $K_{e}$. It is very slightly more energetic than the G100 experiment.

In conclusion, it appears that competing processes are indeed at work, as discussed in the introduction. When the atmosphere is near saturation at low levels, the processes which enhance wave growth dominate. With lower relative humidity, the processes which attenuate wave growth can prevail. It also appears that the enhancement of wave growth depends on having a relative abundance of moisture at low latitudes, whereas the process which attenuates wave growth depends on moisture at mid-latitudes.

To examine the energetics and structure of the life cycles in more detail we concentrate on two of the experiments: the G100 experiment where the wave is strengthened and the G100ST60 experiment where it is weakened, and compare them with the DRY integration. Figure 4 shows the $965 \mathrm{mb}$ temperature at days 5, 7 and 9 for the DRY and the G100 experiments. Areas of diabatic heating are also indicated for the G100 experiment. The first two snapshots show the structure of the wave during its linear growth, while the third shows the wave in the non-linear phase of its life cycle. During the linear growth, the diabatic heating mainly occurs along the warm front, while during the mature phase it occurs ahead of the cold front, close to the occlusion. By day 9 it can be seen that moisture condensation has warmed the latitude band from $40^{\circ}$ to $50^{\circ} \mathrm{N}$ in both cold and warm sectors, but has not substantially altered the phase of the temperature wave. The differing locations for diabatic heating to occur at different stages of the eddy evolution are clearly separated in latitude, and may provide a starting point to investigate the generation of $K_{e}$ in our set of experiments. Figure 5 shows the zonal mean eddy moisture flux convergence averaged over days 5-7 and 8-10 for the G100 and G100ST60 experiments. Shaded areas denote maxima in the diabatic heating. The introduction of a sub-tropical dry zone has a substantial effect on the maximum diabatic heating for both time periods. The effect of introducing a mid-latitude dry zone (not shown) is much less pronounced. The reduction in diabatic heating in the G100ST60 experiment (compared to G100) occurs mainly on the lower-latitude flank of the wave. This is where the most intense diabatic heating occurs in the G100 experiment, especially in the mature phase of wave development. It appears that the G100ST60 experiment lacks the necessary supply of moisture to generate significant precipitation at lower latitudes, ahead of the cold front, as seen in the G100 experiment. This feature has been extinguished, and the precipitation along the warm front is also weakened considerably (not shown). The eddy 

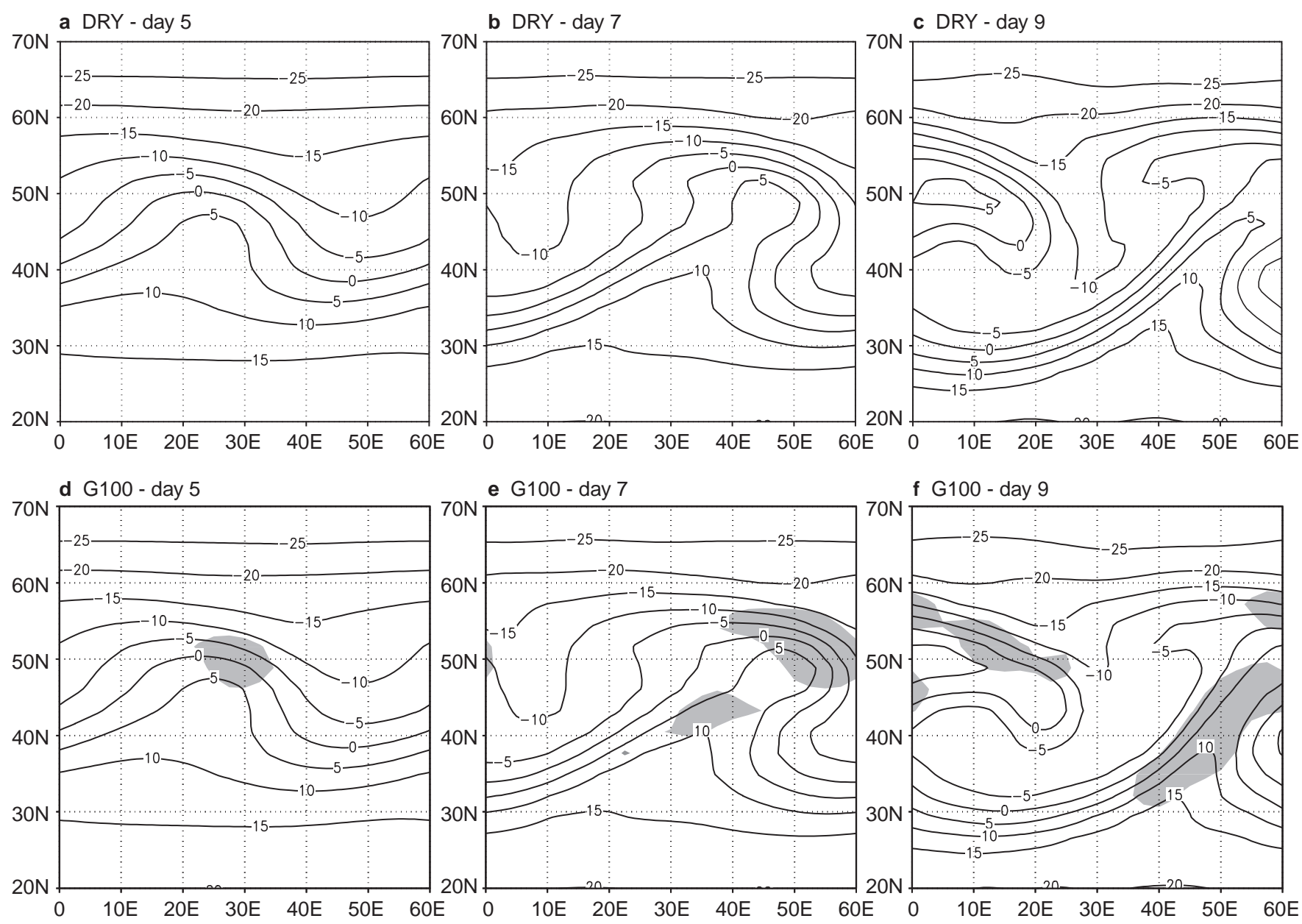

Fig. 4a-f. $965 \mathrm{mb}$ temperature on days 5, 7 and 9 for the DRY experiment (a, b, c) and the G100 experiment (d, e, f). Contours every $5{ }^{\circ} \mathrm{C}$. Shading on $G 100$ denotes areas where the daily mean diabatic heating at this level is greater than $0.5^{\circ} \mathrm{C}$

moisture flux convergence also shown in Fig. 5 is consistent with wave transport of moisture northwards and upwards in all experiments. The moisture contained in sub-tropical air is clearly important, even for condensation heating in mid-latitudes. There is a reasonably good correspondence between the convergence of water and both the location and magnitude of diabatic heating, particularly in the later stage of the life-cycle, when the level of the diabatic heating maximum is raised. In the earlier stage when the heating is at a lower level, the match with the moisture flux convergence is not so good, and a larger proportion of the heating is due to condensation of water present at this location in the initial condition.

We now turn to an analysis of the energetics. The scheme we use was introduced by Pearce (1978) and modified by Blackburn (1983). It has been used recently by Stephenson (1995) to evaluate the role of diffusion in a GCM. Essentially, the available potential energy (APE) is split into three parts. The 'static stability APE', $N_{s}$, measures the departure from an isothermal reference state by the temperature variance in the vertical. $N_{s}$ represents a reservoir of potential energy which is not all strictly 'available', but conversions from $N_{s}$ to other forms can arise if the global mean vertical temperature profile of the atmosphere is altered. The 'zonal APE', $N_{z}$, measures the variance of zonal mean temperature in latitude. The 'eddy APE', $N_{e}$, measures the variance of temperature in longitude. Diabatic sources of $N_{s}, N_{z}$ and $N_{e}$ are referred to as $G_{s}, G_{z}$ and $G_{e}$. The zonal and eddy kinetic energies, $K_{z}$ and $K_{e}$, have their usual meaning. Diabatic sinks of $K_{z}$ and $K_{e}$ due to hyperdiffusion are neglected in the present calculations. Definitions are given in the Appendix.

Figure $6 \mathrm{a}, \mathrm{b}$ shows changes in these types of energy and conversions between them for the DRY experiment. Two periods are shown in the growth phase of the wave, days 5-7 (Fig. 6a) and 8-10 (Fig. 6b). It can be seen that the wave converts $N_{z}$ to $N_{e}$ to $K_{e}$ during both these growth periods, and in the latter period the conversion of $K_{e}$ to $K_{z}$ starts to build more rapidly as the mode reaches nonlinear saturation. There is also a significant conversion from $N_{s}$ to $K_{e}$ at this time.

Moisture affects the energetics in two ways. Firstly a diabatic source is added. Secondly the dynamics are altered, as shown by changes in the conversions. Figure $6 \mathrm{c}, \mathrm{d}$ shows the anomalies from Fig. $6 \mathrm{a}, \mathrm{b}$ for the G100ST60 experiment in which the wave growth is attenuated. Figure 6e,f shows the anomalies for G100, in which wave growth is enhanced. Note first of all that in 
a G100 - day 5 to 7

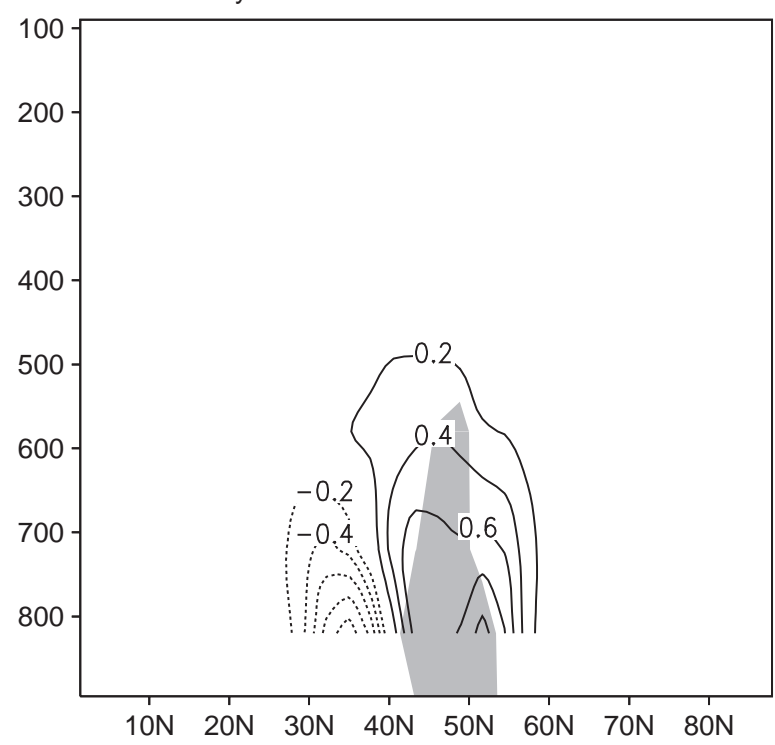

c G100ST60 - day 5 to 7

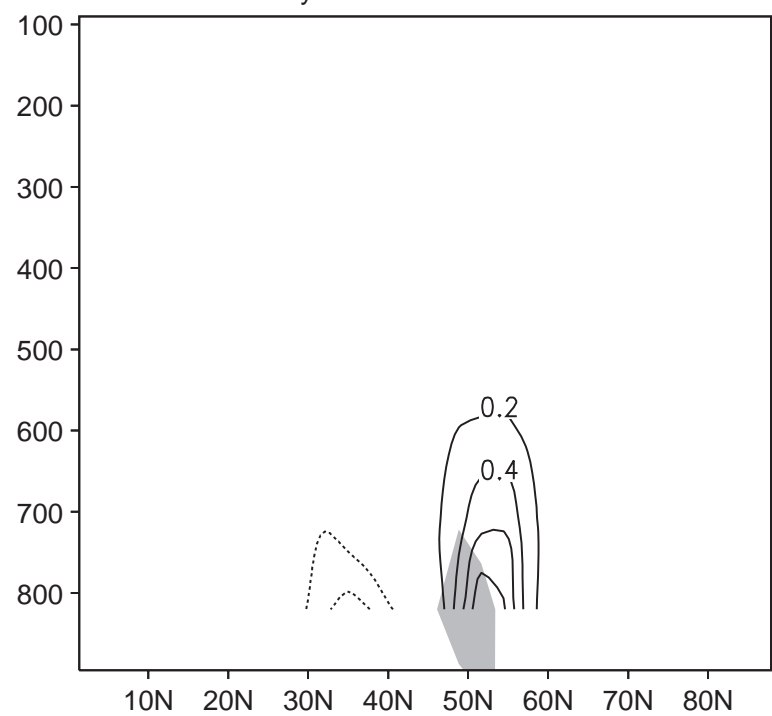

Fig. 5a-d. Zonal mean of moisture flux convergence in units of $\mathrm{g} \mathrm{m}^{-3}$ per day averaged over days 5 to 7 and 8 to 10 for $\mathbf{a}, \mathbf{b} \mathrm{G} 100$ and c, d G100ST60. Contour interval is $0.2(\mathbf{a}, \mathbf{c})$ and $0.4(\mathbf{b}, \mathbf{d})$. Negative contours dashed. Zero contour omitted. Shaded areas denote

both cases there is an early boost to the growth of $K_{e}$. For G100ST60 this trend is then reversed, whereas for G100 it continues.

During the initial linear growth phase the two life cycles look quite similar. There is a diabatic sink of $N_{z}$ (i.e. $G_{z}$ is negative). The latitudinal structure of condensation heating therefore reduces baroclinicity, as postulated already. However, the heating is predominantly at low levels, and there is a source of $N_{s}$ which more than compensates energetically for the loss of $N_{z}$. Also, as the wave grows it organizes the phase structure of the heating to provide a direct diabatic source of $N_{e}$. This source, $G_{e}$, is formed by taking the mass weighted spatial average of its integrand (see Appendix for details). This integrand quantity is shown b G100 - day 8 to 10

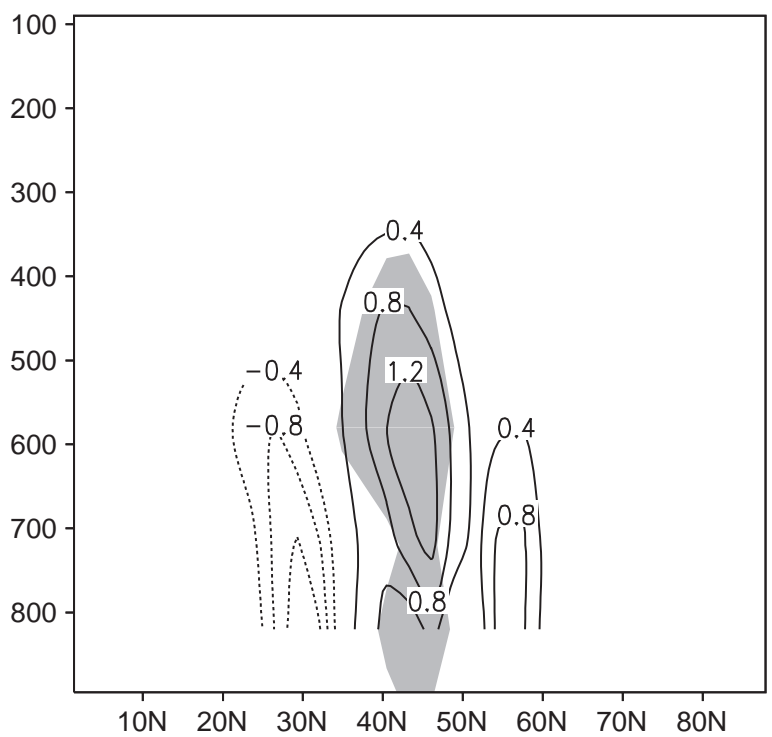

d G100ST60 - day 8 to 10

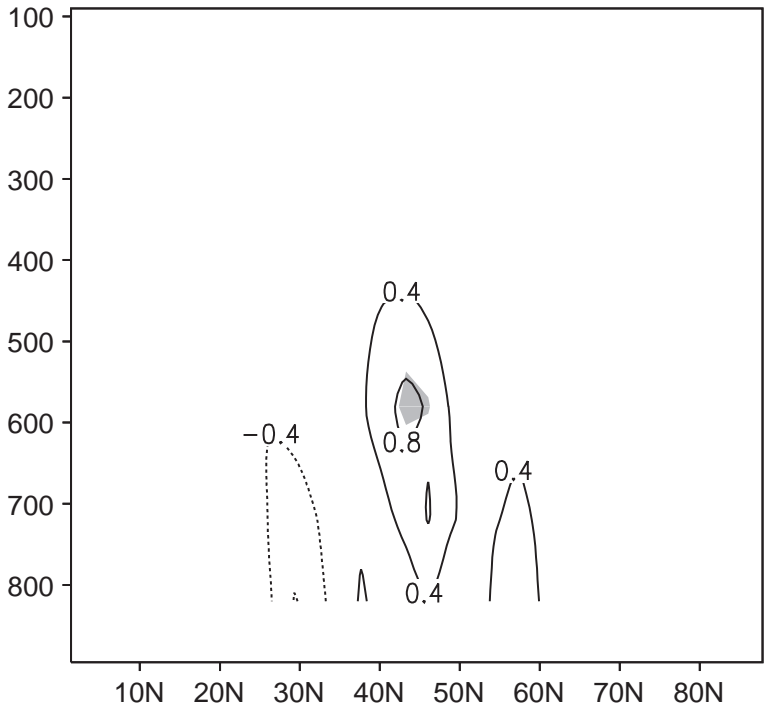

regions where the time-average zonal mean precipitated water exceeds $0.2(\mathbf{a}, \mathbf{c})$ or $0.4(\mathbf{b}, \mathbf{d})$ in the same units. These correspond to maxima in diabatic heating of a $1.2, \mathbf{b} 2.4, \mathbf{c} 0.8$ and $\mathbf{d} 1.2{ }^{\circ} \mathrm{C}$ per day

in Fig. 7a,b. It peaks at low-levels in mid-latitudes where the dry wave is developing, and thus enhances the existing wave. Even at this early stage, between days 5 and 7, there is some difference between the two moist experiments in both the magnitude of $G_{e}$ and structure of its integrand. $G_{e}$ is larger in the G100 experiment $\left(3.8 \times 10^{5} \mathrm{~J} \mathrm{~m}^{-2}\right.$ per day $)$ than in the G100ST60 experiment $\left(1.2 \times 10^{5} \mathrm{~J} \mathrm{~m}^{-2}\right.$ per day). The integrand is slightly more extended to lower latitudes and lower levels in the G100 experiment, as expected given the initial condition on moisture and the diabatic heating.

The conversion from $N_{z}$ to $N_{e}$ is also increased in the early stages of both moist integrations, despite the diabatic drain on $N_{z}$. However, in this case the quantity 

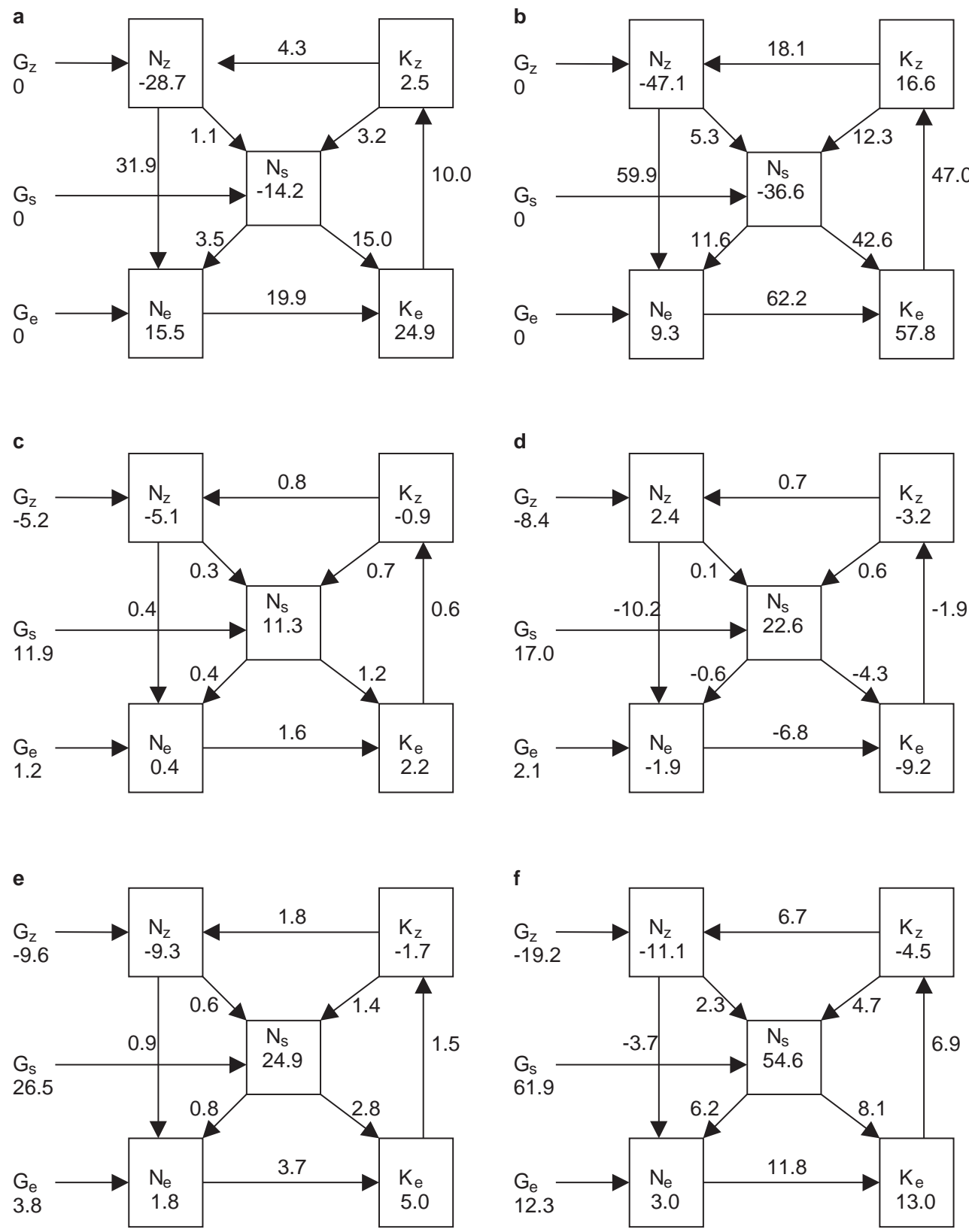

Fig. 6a-f. Energy box diagnostics showing rates of change and conversions between different forms of energy (units $10^{5} \mathrm{~J} \mathrm{~m}^{-2} \mathrm{day}^{-1}$ ) for three life cycle experiments: a, b DRY; $\mathbf{c}, \mathbf{d}$ the anomaly G100ST60-DRY and $\mathbf{c}, \mathbf{f}$ the anomaly G100DRY. Values given are averages over days 5 to $7(\mathbf{a}, \mathbf{c}, \mathbf{e})$ and days 8 to $10(\mathbf{b}, \mathbf{d}, \mathbf{f})$

which integrates to give the conversion shows some cancellation in the vertical. The conversion is effectively enhanced at upper levels but reduced at lower levels. The vertical-latitudinal anomaly structure of the integrand is shown in Fig. 7c,d for the (G100ST60-DRY) and (G100-DRY) experiments (see the Appendix for definitions). To understand the reasons for the verticalmeridional structure of the change in this conversion integrand we need to consider its definition as given in the Appendix. The conversion depends on the product of temperature flux with temperature gradient. We assume, based on the similarity of the DRY and G100 runs in Fig. 4, that the flux is relatively unchanged. We assume also that the horizontal and vertical fluxes reinforce one another. The effect of changes in temperature gradient alone on the conversion can then be evaluated. These changes in temperature gradient are readily deduced from the position of the diabatic heating. The meridional temperature gradient should be increased to the north of the heating and decreased to the south. The gradient with respect to pressure should be increased above the heating and decreased below it. Thus the effect of changing temperature gradients, brought on by diabatic heating shown in Fig. 5, leads to the observed distribution of the quantity which integrates to give the conversion from $N_{z}$ to $N_{e}$. This quantity, shown in Fig. 7d,e, shows a fair degree of cancellation in the vertical. The conversion could therefore be quite sensitive to the distribution of diabatic heating, and its spatial relationship with existing temperature gradients. The integrands shown in Fig. 7 for the two experiments have similar anomaly structures but the magnitude is again different. In this initial, linear stage the enhancement at upper levels only just out- 

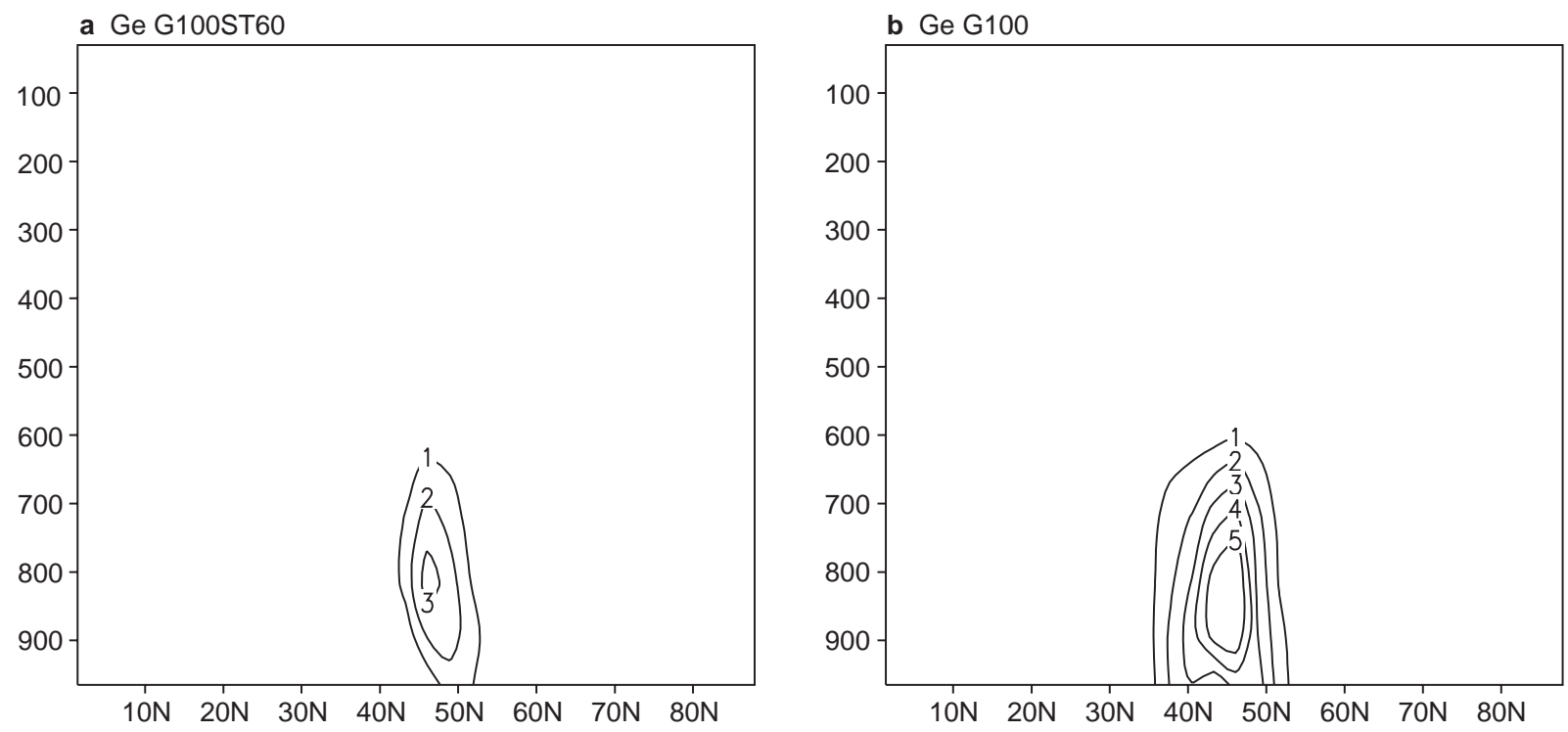

c $\mathrm{C}(\mathrm{Nz}: \mathrm{Ne})(\mathrm{G} 100 \mathrm{ST} 60-\mathrm{DRY})$

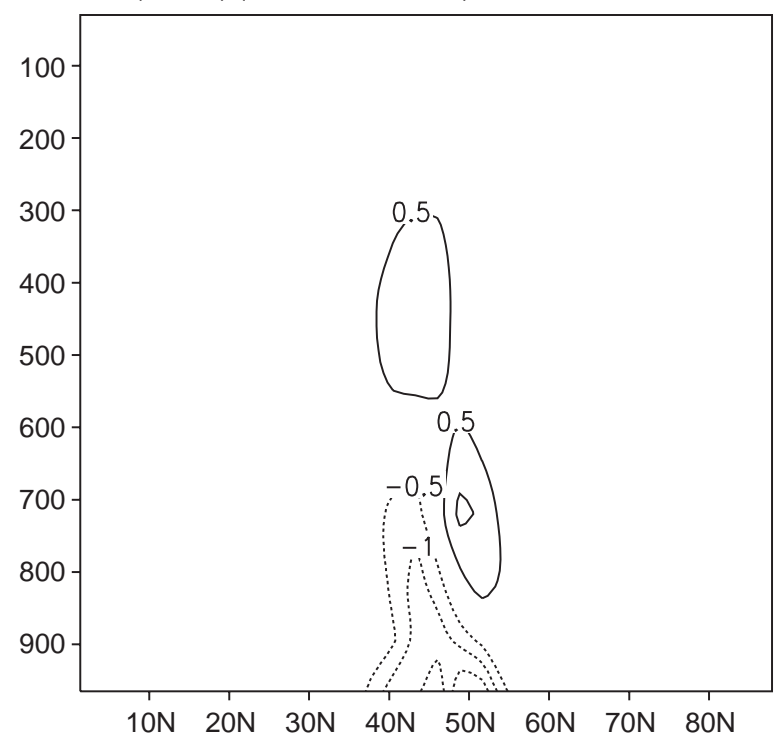

d $\mathrm{C}(\mathrm{Nz}: \mathrm{Ne})(\mathrm{G} 100-\mathrm{DRY})$

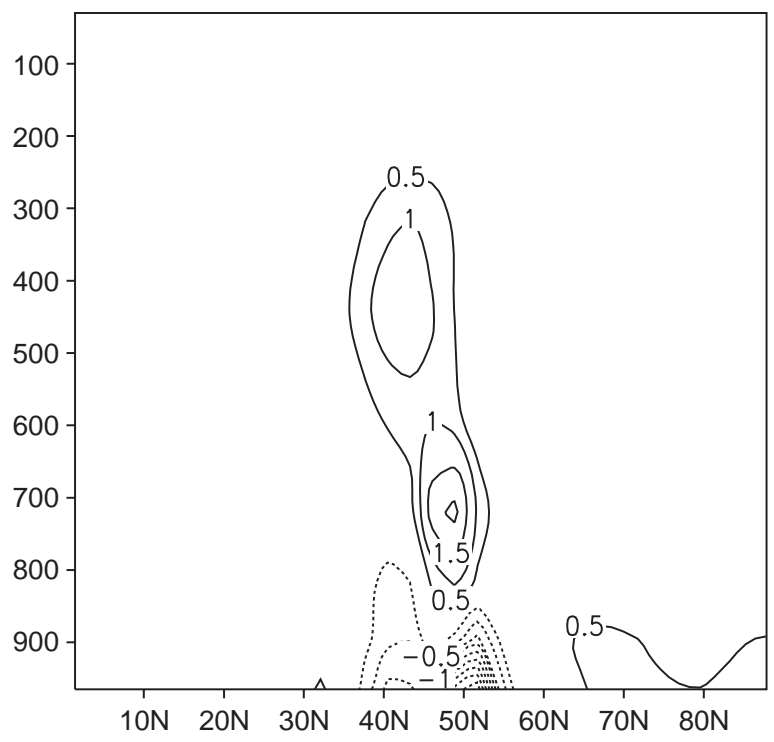

Fig. 7a,b The average spatial distribution over days 5 to 7 of the integrand of the diabatic source term $G_{e}$, for a G100ST60 and b G100. Contours every $10^{5} \mathrm{~J} \mathrm{~m}^{-2}$ per day. c, d. The average spatial distribution over days 5 to 7 of the integrand of the conversion from

$N_{z}$ to $N_{e}$ for $\mathbf{c}$ the anomaly G100ST60-DRY and d the anomaly (G100-DRY). Contours every $0.5 \times 10^{5} \mathrm{~J} \mathrm{~m}^{-2}$ per day. Zero contour omitted. Negative contours dashed

weighs the reduction at lower levels, where the dry mode is growing, to give a net increase in the conversion from $N_{z}$ to $N_{e}$ in both cases.

The situation is therefore more complicated than a competition between a diabatic supply of $N_{e}$ and a diabatic depletion of $N_{z}$, as envisaged in the introduction. In addition there is a change in the conversion from $N_{z}$ to $N_{e}$ which comes about via a reduction in its integrand at low levels, coupled with an increase aloft. This cancellation can be understood in terms of the change in vertical and meridional temperature gradients brought about by diabatic heating. The possibility therefore exists that the future development of the lifecycle is quite sensitive to the form of the diabatic heating.

The overall structural similarity between the two moist life cycles in days 5-7 implies that the ensuing difference between them must be due to a finite amplitude development. In the real atmosphere this need not be the case, as a suitably configured preexisting disturbance may be able to tap the same energy source as the finite amplitude development. However, in the case of our normal mode life cycles a sensitivity emerges in days 8-10 that leads ultimately to a reduction in $K_{e}$ in one case (G100ST60) and an increase in $K_{e}$ in the other (G100). Figure 8 shows the latitude-height structure of the quantities which integrate to give the conversions from $N_{z}$ to $N_{e}$ and $N_{e}$ to $K_{e}$ for days 8-10 of the DRY experiment. The effect of moisture on these quantities is then shown in Fig. 9, in the form of 
a $\mathrm{C}(\mathrm{Nz}: \mathrm{Ne}) \mathrm{DRY}$

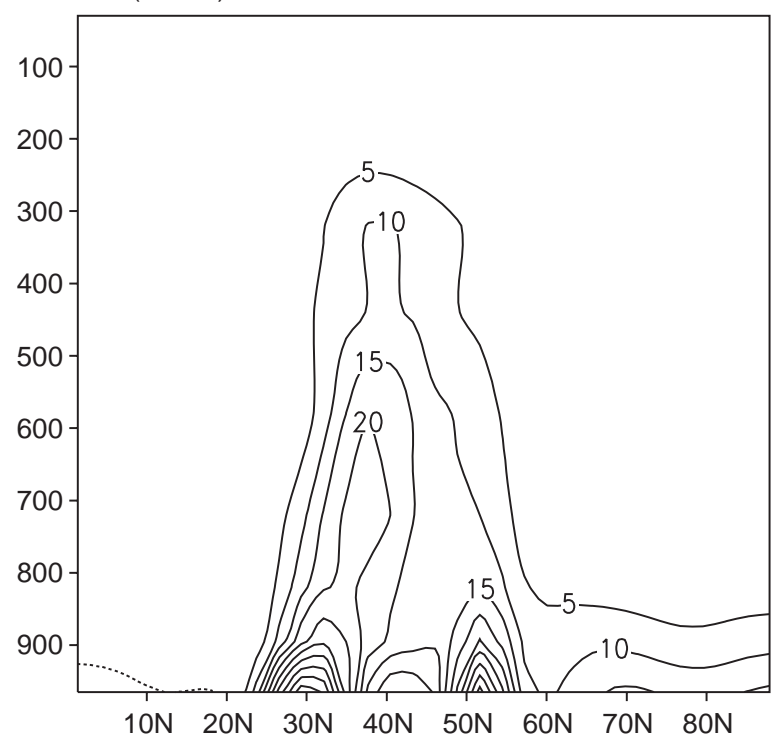

b $\mathrm{C}(\mathrm{Ne}: \mathrm{Ke}) \mathrm{DRY}$

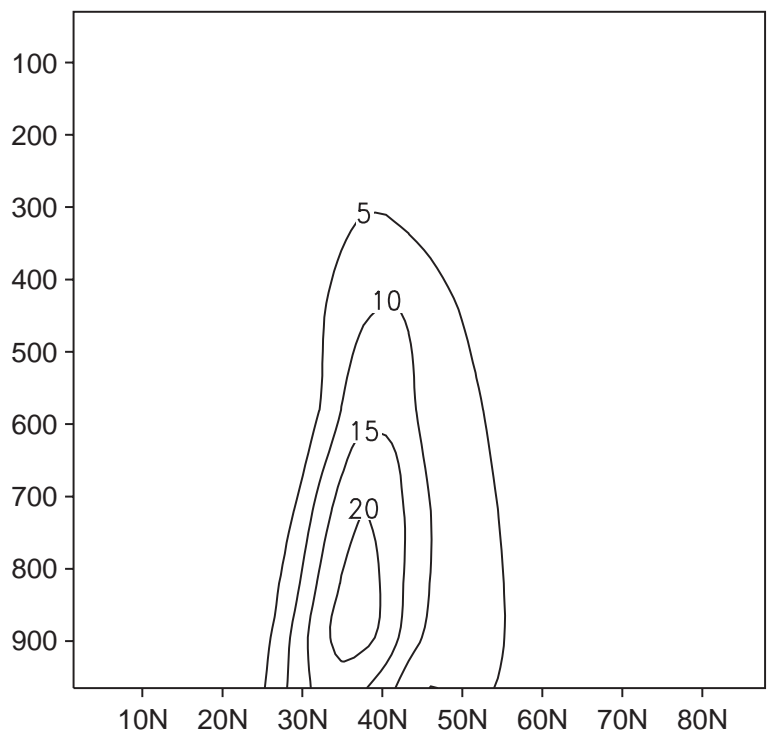

Fig. 8a, b. The average spatial distribution over days 8 to 10 of the integrand of the conversion from a $N_{z}$ to $N_{e}$ and $\mathbf{b} N_{e}$ to $K_{e}$ for the DRY life cycle. Contours every $5 \times 10^{5} \mathrm{~J} \mathrm{~m}^{-2}$ per day

anomaly plots for the G100ST60 and G100 experiments. The integrand of $G_{e}$, the diabatic source of $N_{e}$, is also shown in Fig. 9 for this time period.

For the G100ST60 experiment the growth of $N_{e}$ is still enhanced by the diabatic source $G_{e}$, with an integrand structure close to that of $N_{e}$ in the main (dry) development. However, there is relatively little increase in this source to sustain the growing wave. The low-level contribution to the conversion from $N_{z}$ to $N_{e}$ is drastically reduced. The consequent reduction in $N_{e}$ is passed on to $K_{e}$ leading to a weakening of the wave.

For the G100 experiment, the growth of $N_{e}$ is much more strongly enhanced by $G_{e}$, particularly by contributions from the low-level sub-tropics. Furthermore, the reduction in the conversion from $N_{z}$ is not as strong because, even though the low-level signature is still present, there is a development at upper levels and higher latitudes which is able to compensate. The resulting increase in $N_{e}$ is passed on to $K_{e}$ as an upper level development.

The mechanism of differing growth in the two cases can be understood in terms of the initial condition on relative humidity, the subsequent transport of moisture and thence the diabatic heating. Initially (days 5-7) both life cycles show diabatic heating, although it is greater in the G100 experiment. Indeed, both life cycles have a heating structure which gives an initial boost to $K_{e}$, as $G_{e}$ feeds $N_{e}$. At the same time, $N_{z}$ is depleted, but its conversion to $N_{e}$ is still increased. However, by days 8-10 the G100ST60 experiment is not able to sustain the low-latitude precipitation and only the G100 experiment can contribute enough diabatic heating to maintain positive values of the conversion from $N_{e}$ to $K_{e}$. Conversions from $N_{s}$ to $K_{e}$ are also significant but they have a very similar structure to conversions from $N_{e}$ (although the diabatic sources of $N_{s}$ are very large in both cases they are largely absorbed in this reservoir, and do not become dominant in the wave dynamics). Anomalies in the conversions from $K_{e}$ to $K_{z}$ at this stage simply reflect the amount of $K_{e}$, and have an integrand structure similar to the DRY experiment, although a little more amplified at upper levels in the G100 case.

In summary, we have found that the presence of moisture in the initial condition leads to diabatic heating which provides a source of eddy available potential energy. It also leads to a depletion of zonal available potential energy. However, the effect it has on the conversion from zonal to eddy available potential energy is more complicated, as there is cancellation between upper and lower levels. If the sub-tropics are relatively dry, the reduction in the conversion at low levels can dominate, and slow down wave development. If moisture is plentiful in the sub-tropics, this effect can be overwhelmed as the direct source of eddy available potential energy $G_{e}$ remains strong. To some extent, both these processes are present whatever the initial condition on humidity, because moisture is transported northwards by the developing wave. In this way subtropical moisture can contribute mid-latitude condensation, but the net effect of subtropical moisture is still to boost the wave through the way it affects both $G_{e}$ and the conversion from $N_{z}$ to $N_{e}$. The balance between the competing processes is affected by the initial condition. In cases

Fig. 9a-f. The average spatial distribution over days 8 to 10 of the integrand of: a, b the source, $G_{e}$ for G100ST60 and G100, (contours every $10^{5} \mathrm{~J} \mathrm{~m}^{-2}$ per day), c, $\mathbf{d}$ the conversion from $N_{z}$ to $N_{e}$ for the anomalies G100ST60-DRY and G100-DRY and e, $\mathbf{f}$ the conversion from $N_{e}$ to $K_{e}$ for the anomalies G100ST60-DRY and G100-DRY (contours every $2 \times 10^{5} \mathrm{~J} \mathrm{~m}^{-2}$ per day). Zero contour omitted. Negative contours dashed 

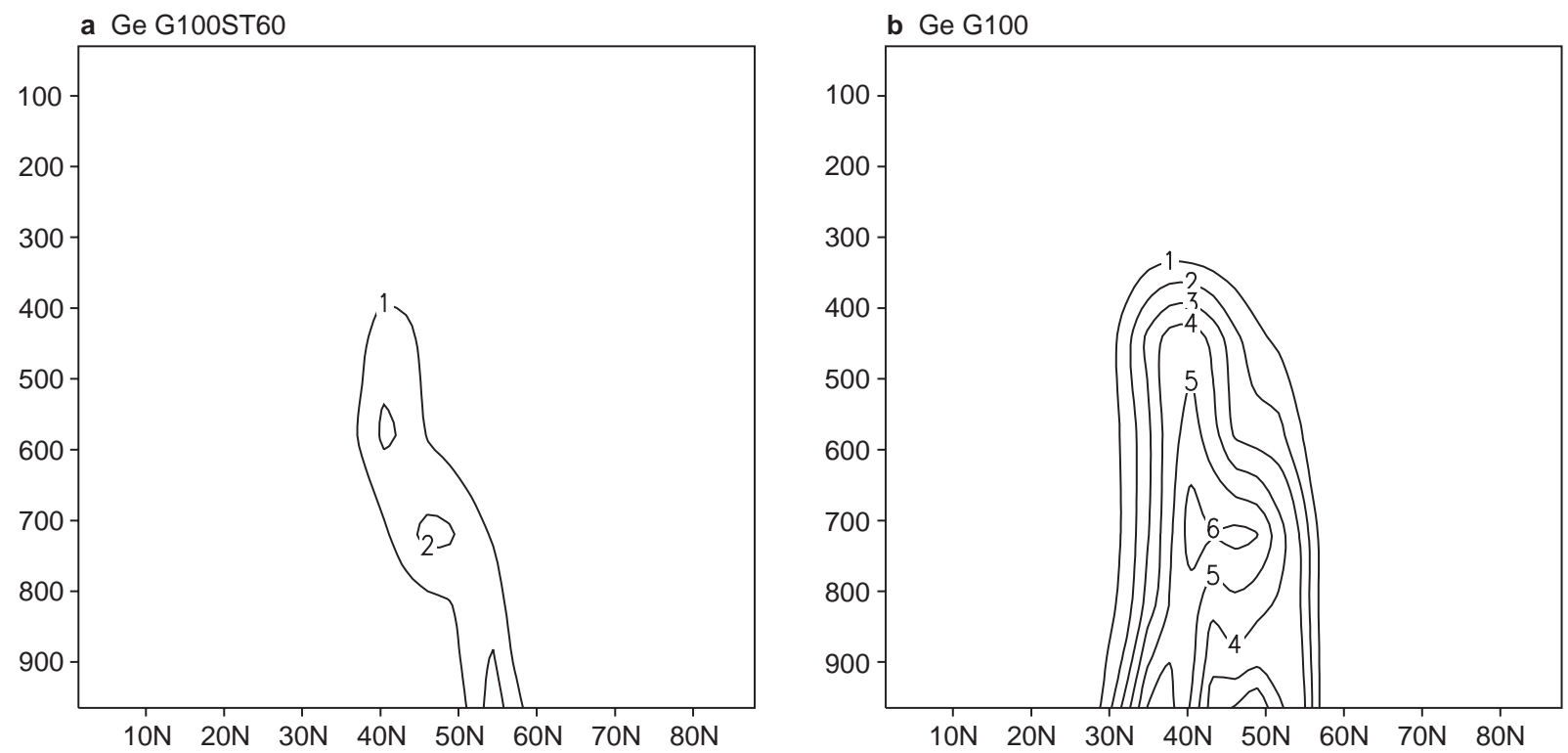

c $\mathrm{C}(\mathrm{Nz}: \mathrm{Ne})(\mathrm{G} 100 \mathrm{ST} 60-\mathrm{DRY})$

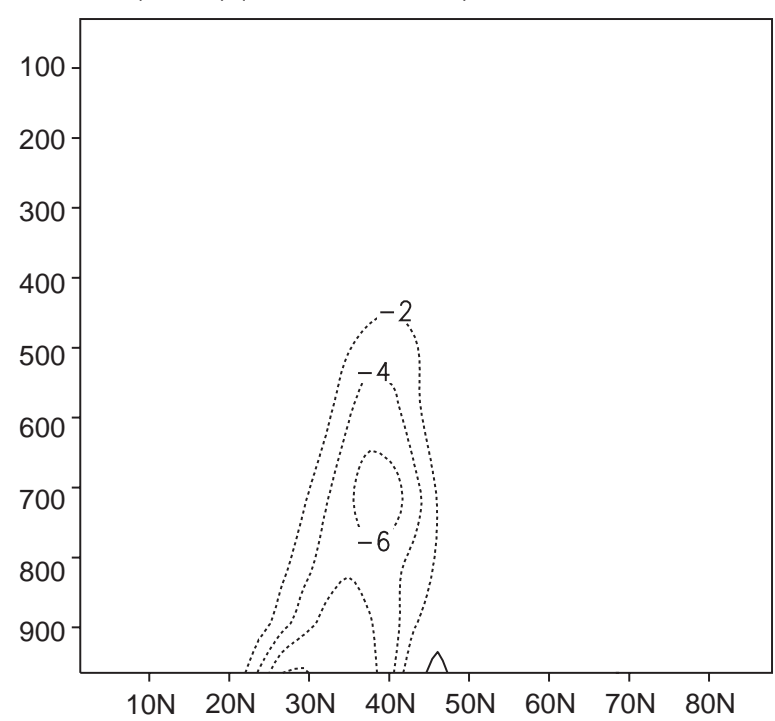

d $\mathrm{C}(\mathrm{Nz}: \mathrm{Ne})(\mathrm{G} 100-\mathrm{DRY})$

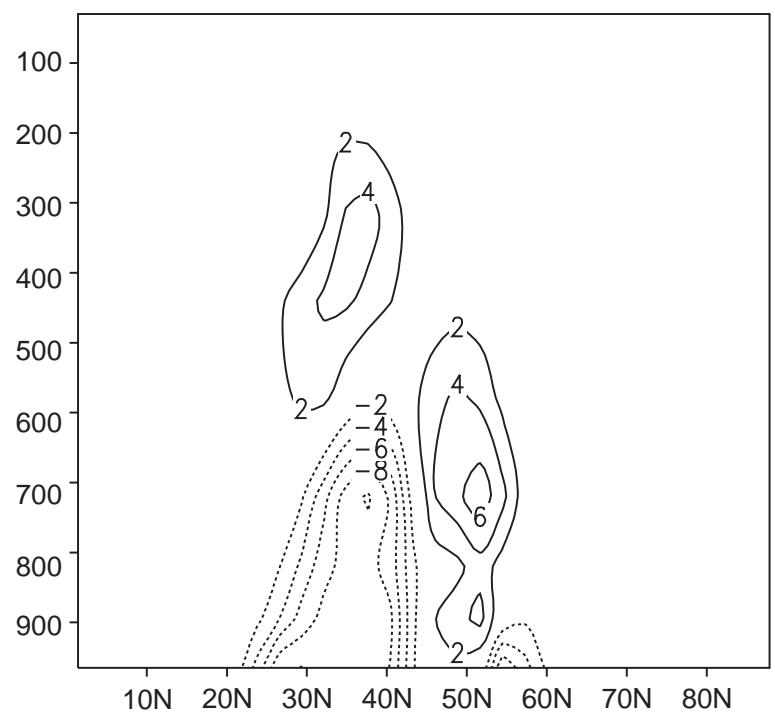

e $\mathrm{C}(\mathrm{Ne}: \mathrm{Ke})(\mathrm{G} 100 \mathrm{ST} 60-\mathrm{DRY})$

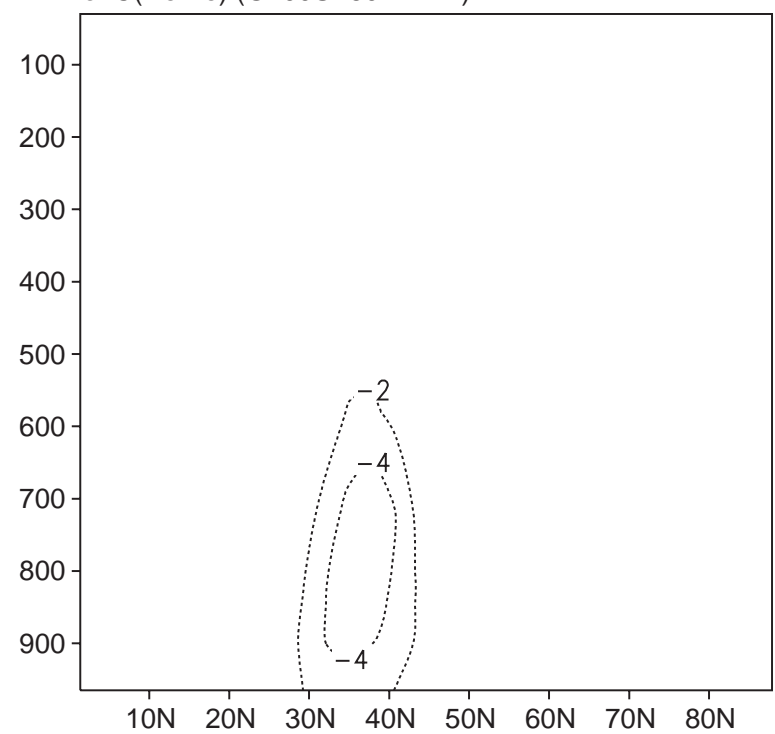

f $\mathrm{C}(\mathrm{Ne}: \mathrm{Ke})(\mathrm{G} 100-\mathrm{DRY})$

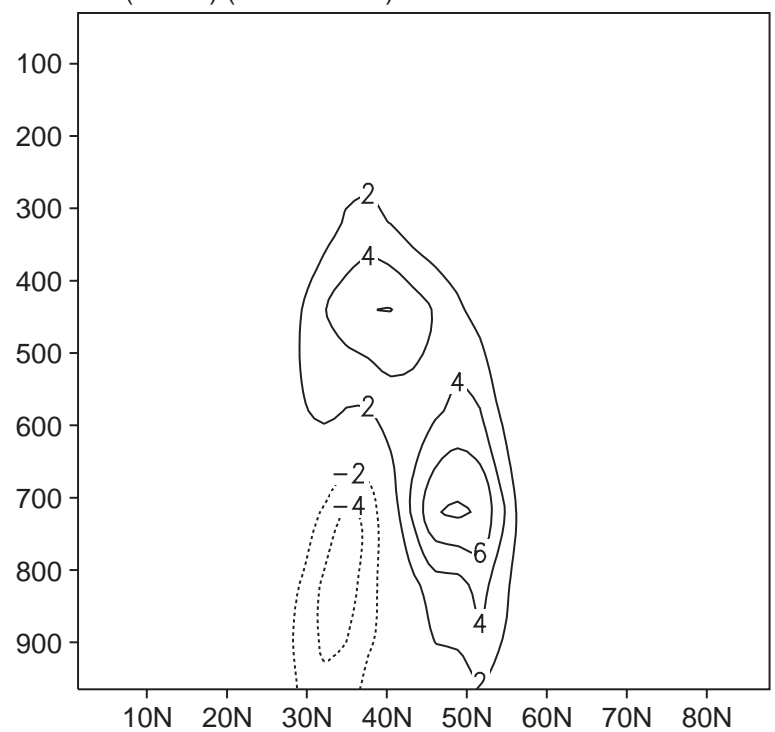


where $G_{e}$ is big enough and the reduction in conversions from $N-z$ small enough, the wave can develop so as to extract further energy from the zonal mean temperature structure in the upper troposphere. This gives rise to a stronger development aloft and leads to increased generation of eddy kinetic energy.

\section{Large-scale precipitation, surface fluxes and moist convection}

\subsection{Experimental design}

The experiments described in this section repeat those of the last section with the addition of two further physical parametrizations: surface fluxes into the boundary layer and moist convection. The boundary layer parametrization includes surface fluxes of momentum, heat and moisture. They are defined in terms of the following bulk quantities:

$$
\begin{aligned}
& \vec{\tau}=\overline{\rho_{s}} c_{d}|\overline{\mathbf{v}}| \mathbf{v} \\
& \mathscr{H}=\overline{\rho_{s}} c_{h}\left[|\mathbf{v}|+\mathscr{A}\left(\frac{\triangle \theta}{\bar{\theta}}\right)^{\frac{1}{2}}\right] \triangle \theta \\
& \mathscr{Q}=\overline{\rho_{s}} c_{q}\left[|\mathbf{v}|+\mathscr{A}\left(\frac{\triangle \theta}{\bar{\theta}}\right)^{\frac{1}{2}}\right] \triangle q
\end{aligned}
$$

where $c_{d}$ is set to $10^{-3}$, appropriate for oceanic conditions, $c_{q}=c_{h}=c_{d} / 5, \overline{\rho_{s}}$ is the horizontally averaged density, and $\mathscr{A}$ is zero in stable conditions and is set to $500 \mathrm{~m} \mathrm{~s}^{-1}$ in unstable conditions. $\triangle \theta$ and $\Delta q$ are the differences in potential temperature and specific humidity between the top of the lowest model layer and the surface (surface values of $\theta$ and $q$ are fixed and the surface is assumed to be ocean). The tendencies of horizontal velocity, temperature and specific humidity are modified, in the lowest model layer only, proportional to $\vec{\tau}, \mathscr{H}$ and $\mathscr{Q}$ respectively in a way equivalent to a boundary layer with depth equal to the depth of the lowest model layer.

Sub-grid scale convection is parametrized using a simplified adjustment scheme based on Betts (1986). This scheme relaxes towards a sub-saturated moist adiabatic profile for deep convection and to a mixing line in the presence of a low-level capping inversion. It complements the large-scale rain parametrization used in the previous section by representing the effect of latent heating in convective vertical motion which is not resolved by our model.

\subsection{Results}

The effect of boundary layer drag alone has been investigated by repeating the DRY experiment and two of the large-scale rain experiments from the previous section, but including just the low-level momentum tendency given earlier. Figure 10a shows the time development of $K_{e}$ for six life cycles. The three lower curves are the DRY, G100 and G100ST60 results repeated from the previous section for comparison. The three upper curves have the same initial conditions, but are now subject to boundary layer drag in addition to the large scale rain parametrization. Initially the drag acts directly to drain the $K_{e}$ of the developing wave, slowing its exponential growth. However, the drag also acts on the background surface wind which was initially a

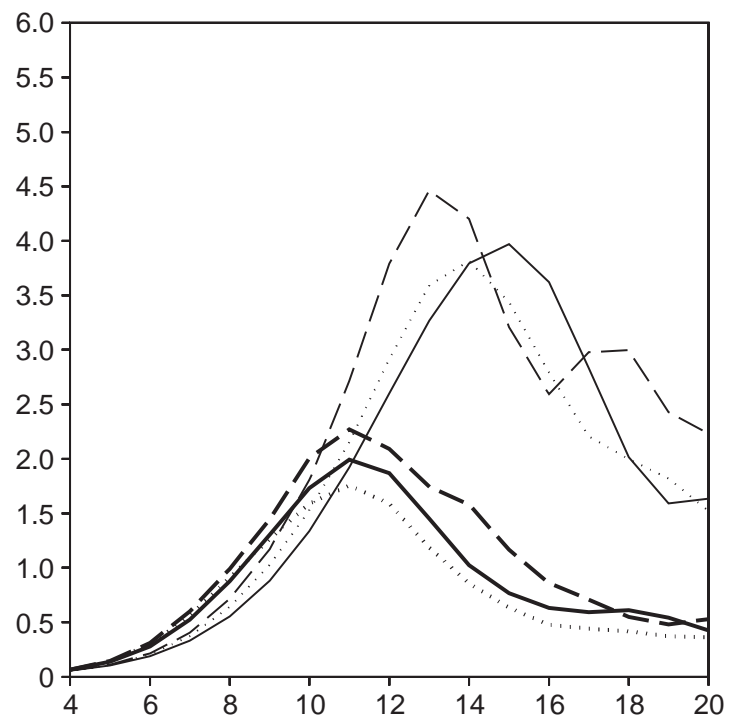

b

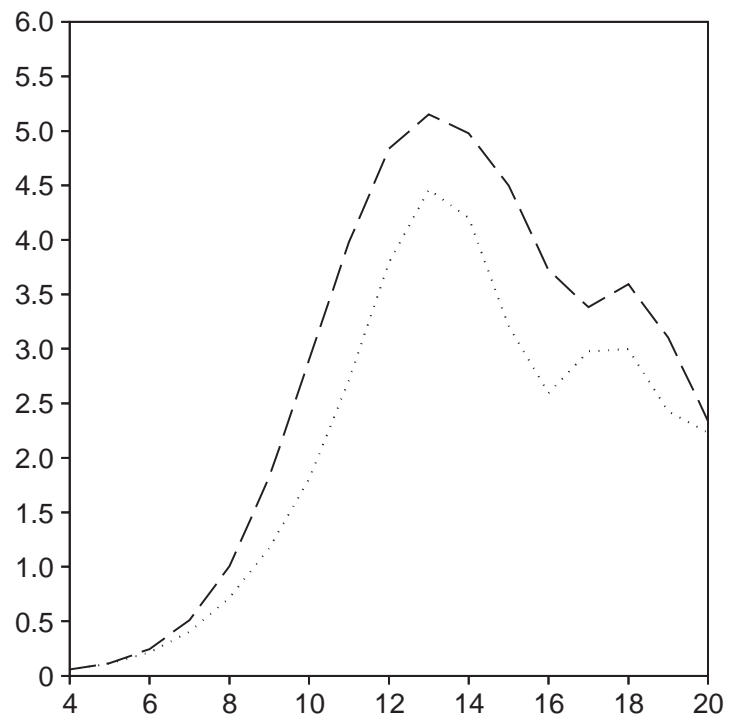

Fig. 10. a Time development of eddy kinetic energy, $K_{e}$ as in Fig. 3, for 6 life cycles. Heavy curves have just the large-scale rain parametrization. Light curves have boundary layer drag added. Solid curves are DRY, dotted curves G100ST60, dashed curves G100. b As a but for G100ST60 (dotted) and G100 (dashed) with surface fluxes of heat and moisture and deep convection added to the parametrizations. Values plotted in units of $10^{5} \mathrm{~J} \mathrm{~m}^{-2}$ 
non-zero in these life cycles. It appears that this barotropic component of the initial condition was acting to inhibit wave growth (separate experiments with no drag and no initial surface will confirm this interpretation). When the surface wind is gradually reduced by drag, the peak $K_{e}$ is approximately doubled. In energetic terms, the transfer from $N_{z}$ to $N_{e}$ has been increased because of a reduction in barotropic shear. James and Gray (1986) introduced the idea of a 'barotropic governor' on normal mode development. They envisaged its appearance as a consequence of nonlinear eddy-mean flow interaction. In our case its disappearance is a consequence of explicit friction. The overall effect of boundary layer drag is therefore a delay in the lifecycle and an amplification of peak $K_{e}$. The delay is particularly marked in the DRY case.

However, even though there has been a major alteration of the basic state, the sensitivity of the result to initial moisture distributions remains the same. Condensation heating due to large-scale rain has still enhanced the final wave amplitude for G100 and reduced it for G100ST60, and it has done so by approximately the same amount as before. The conclusion is therefore that the sensitivity to the initial moisture distribution found in the previous section is robust to the effect of surface friction and the subsequent modification of the background flow and the structure of the wave.

To complete the study we add the rest of the parametrizations given in Sect. 3(.1): surface fluxes of heat and moisture and deep convection. We repeat the two moist experiments, G100 and G100ST60 again. The results are given in Fig. 10b. It can be seen that there is a slight further enhancement due to the additional parametrizations. In fact this enhancement now means that both moist experiments are now more energetic than the dry experiment. Although the possibility that the initial condition on moisture can actually decrease the peak $K_{e}$ has now been removed, we still see that the difference between the two moist runs is similar to what it was in the absence of deep convection and surface fluxes. This may be viewed as surprising since the zonal mean basic state has now been modified within the life-cycle not only by the action of the wave, but also by sources of momentum, heat and moisture, and parametrizations which can act independently of the wave's existence, potentially diminishing the importance of differences in initial conditions. However, the fact that in our case such sensitivity is robust suggests that between the many processes which could interfere with the dynamics of baroclinic waves, large-scale precipitation has an important role, whose effects on the eddies depend substantially on the initial conditions of moisture.

\section{A doubled $\mathrm{CO}_{2}$ experiment}

All life-cycle experiments described so far have used the same initial conditions on winds and temperature as given in Fig. 1. This initial condition was taken from the zonal mean climate of a 'control' integration of a GCM.
To set the work in the context of climate change, as discussed in the introduction, we include here a brief section in which we examine the life-cycle ensuing from the ' $2 \times \mathrm{CO}_{2}$ ' simulation with the same GCM (Mitchell et al., 1990). The differences in the initial conditions are archetypal for this type of study. The zonal mean temperature has increased throughout the troposphere, but particularly at low levels/high latitudes and at upper levels/low latitudes. The zonal mean baroclinicity is therefore reduced at low levels and increased at upper levels, by up to $3{ }^{\circ} \mathrm{C}$ over $30^{\circ}$ latitude. The zonal mean jet is shifted northwards by about $5^{\circ}$ and the zonal mean wind at the surface is increased by up to $5 \mathrm{~m} \mathrm{~s}^{-1}$. These differences can be seen in detail in Figs. 1 and 3 of Hall et al. (1994). Initial conditions on zonal mean relative humidity are now also taken from the GCM 'control' and ' $2 \times \mathrm{CO}_{2}$ ' integrations as appropriate. Although there is a general increase in specific humidity of $15 \%$ to $30 \%$ on doubling $\mathrm{CO}_{2}$, it is mainly a warming effect as the relative humidity shows little change.

We have followed the same sequence of successively adding parametrizations as in the rest of the study, and applied it to 'control' and ' $2 \times \mathrm{CO}_{2}$ ' life cycle experiments, initiated with the appropriate wave number 6 normal mode perturbations. The results for $K_{e}$ are given in Fig. 11 for all the lifecycles. Solid curves denote control and dashed curves ' $2 \times \mathrm{CO}_{2}$ '. The thick solid curve is just the 'DRY' curve again. It represents our dry 'control' run. Its ' $2 \times \mathrm{CO}_{2}$ ' counterpart (thick dashed curve) is considerably weaker, reaching only two thirds the peak $K_{e}$. The life-cycle is weakened for two reasons. Firstly the reduced baroclinicity in the ' $2 \times \mathrm{CO}_{2}$ ' climate leads to a reduced linear growth rate for the wave. Secondly the increased surface wind attenuates the wave growth still further, as discussed in Sect. 3.

Addition of large-scale rain alone, as in Sect. 2, weakens both life cycles by approximately the same proportion. This can be seen in the lighter two curves of Fig. 11a. Examination of the energetics reveals a similar development to that of the G100ST60 experiment in Sect. 2. Indeed, both GCM relative humidity profiles resemble the G100ST60 profile to a reasonable approximation. The attenuation effect seen in the G100ST60 therefore occurs in both experiments, and does not contribute much to the difference between them.

The two bold curves in Fig. 11b correspond to the addition of boundary layer drag as in the beginning of Sect. 3. The delay and amplification explained there is seen again here. The two curves are somewhat closer now in terms of their exponential growth rate, indicating that some of the attenuation in the ' $2 \times \mathrm{CO}_{2}$ ' case was due to the surface wind in the initial condition. Separate hybrid experiments in which we have initialized the model using a barotropic wind taken from the 'control' and the baroclinic wind from the ' $2 \times \mathrm{CO}_{2}$ ' basic states (not shown) also confirm this. This, then is perhaps a more realistic 'greenhouse' simulation, as the surface westerlies in the GCM could be viewed as a consequence of eddy activity, and therefore not a desirable feature of the initial condition for a life-cycle experiment. However, the $2 \times \mathrm{CO}_{2}$ signature remains weaker despite the 
a

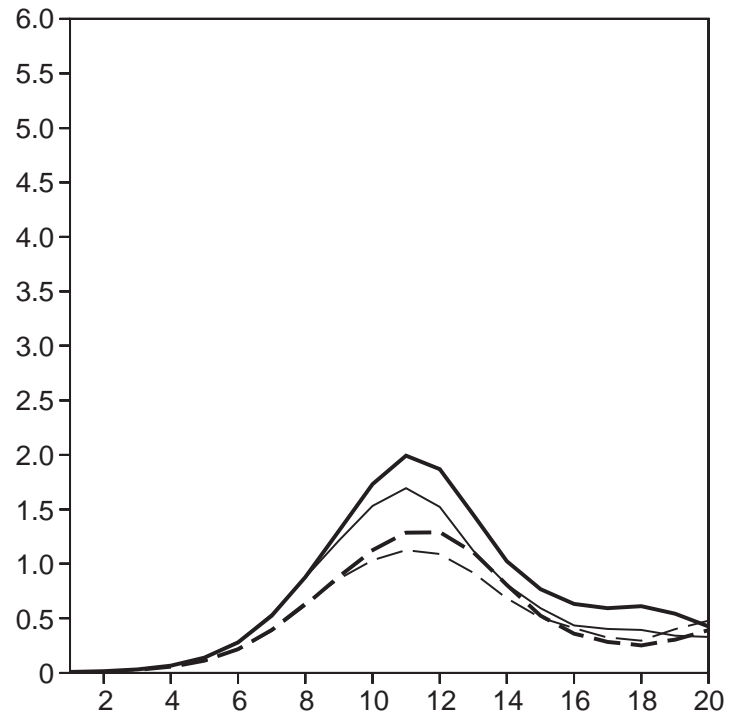

b

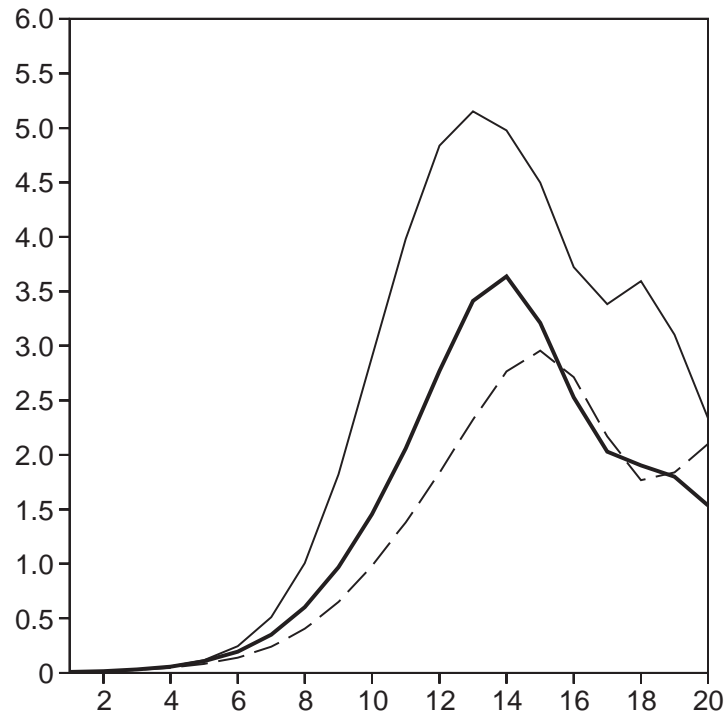

Fig. 11a, b. Time development of eddy kinetic energy, $K_{e}$ for life cycle experiments based on 'control' (solid lines) and ' $2 \times \mathrm{CO}_{2}$ ' (dashed lines) basic states. a DRY lifecycles (heavy curves) and life cycles with just large-scale rain included (lighter curves). b life cycles with large- scale rain and boundary layer drag (heavy curves) and with added surface heat and moisture fluxes and deep convection (lighter curves). Values plotted in units of $10^{5} \mathrm{~J} \mathrm{~m}^{-2}$ increased moisture supply available to the large-scale condensation scheme through the initial condition.

Finally, the largest amplitude pair of curves in Fig. 11b include surface fluxes of heat and moisture and deep convection, as in the last part of Sect. 3. This results in considerable further amplification of the waves over the previous cases. The gap between 'control' and ' $2 \times \mathrm{CO}_{2}$ ' has now widened. The boost given to the amplitude of the wave has not favoured the ' $2 \times \mathrm{CO}_{2}$ ' experiment. The initial condition on relative humidity has had a large influence on the result. In both cases, the initial relative humidity was configured in such a way that large-scale condensation would reduce wave amplitude. It is possible that this effect is exaggerated in the warmer ' $2 \times \mathrm{CO}_{2}$ ' simulation where there is more moisture available, especially in the case where the wave reaches a relatively large amplitude.

\section{Conclusion}

We have attempted to shed light on the question of whether the presence of moisture strengthens or weakens transient activity in the mid-latitudes. The approach adopted has been to study a single eddy life-cycle with a simple parametrization of condensation heating. In diagnosing the results we have concentrated on the exchange between different types of energy. The simplicity of the approach precludes any bold general conclusions. We have, however, identified the range of initial conditions over which the sign of the response changes, and this is a useful thing to know.

Emphasis has been given to variations in the initial condition on relative humidity. For most of the experiments the initial temperature profile was unchanged, so this is equivalent to studying sensitivity to variations in initial water vapour content. Using relative humidity merely draws attention to the closeness to saturation. The two moist cases in Sect. 2, G100 and G100ST60, are highly idealized. In fact both are a bit too moist at low levels in mid-latitudes, while at low latitudes G100 is too moist and G100ST60 too dry. They were picked to illustrate their differing developments. A 'most realistic' case would conform to something like a 'G90ST80' experiment and would lie on the turning point of the sensitivity described in Sect. 2. It may be that the resolution of this issue therefore lies outside the scope of this study. On the other hand, it may be that with realistic amounts of atmospheric moisture, a moist 'storm track' is about as energetic as a dry one, an intriguing coincidence.

Variations in moisture available for condensation arise for a variety of reasons. There are considerable variations in both specific and relative humidity with longitude, so the behaviour of a mid-latitude system may depend on where it was when it started to grow. It should also be noted that some systems are much more efficient at entraining subtropical air than others. The type of system which emerges can depend on easily realisable differences in the ambient flow (Thorncroft et al., 1993). In comprehensive GCM simulations, the mean humidity can vary greatly from one GCM to another, and GCMs often underestimate this quantity (see Table 2 of Gutowski et al., 1995). The ways in which GCMs treat condensation heating can be a further source of variation.

The sensitivity illustrated by our simple experiments reveals a degree of uncertainty about the role of moisture in the existing climate which makes prediction a precarious business. If one is to speculate on the 
possible role of moisture in climate change or climate variability pertaining to mid-latitude transients, then one should note that competing effects are at work. With this in mind, it may be better to try to identify changes in distribution than in amplitude. When $\mathrm{CO}_{2}$ is increased, the primary signal is of increased moisture everywhere. This may not mean very much. Competing effects that cancelled in the control climate can cancel again in the perturbed climate. The tendency for 'global' warming to increase with latitude may tip the balance slightly in favour of weaker transients as the midlatitude moisture content is boosted. But this effect has not been demonstrated separately from the primary effect of reduced baroclinicity on the dry dynamics, which it tends to dominate in our ' $2 \times \mathrm{CO}_{2}$ ' life cycles.

The eddy life-cycle approach to climate sensitivity studies is admittedly unable to deliver the full picture. Variations with longitude in the background flow and temperature field are ignored and yet they can be very important for the eddies, as illustrated by Hall et al. (1994). It should also be noted that the normal mode structures studied here are by no means unique, and disturbances with other structures can be realised. However, all possible growing structures of similar spatial scale draw their energy from the same source, the potential energy available for baroclinic conversions. In this study we have concentrated on processes which can alter these conversions, so it is entirely possible that our principle findings are more generally applicable. A single eddy life-cycle can be a useful test bed if it points to sensitivities, and the processes that give rise to these sensitivities can be diagnosed. In this work we have highlighted a specific sensitivity which may be important in understanding climate response. The insight gained from this type of exercise can feed back into the work of planning and diagnosing more comprehensive simulations.

Acknowledgements. We thank Cath Senior for providing the output from the UK Meteorological Office GCM simulations. We also thank the two reviewers for comments which were very helpful in improving the manuscript. This work was done mainly at Reading University, UK, where V. Pavan was supported by EC grant EV5V-CT94-5218 and N. M. J. Hall by EC grant EV4C.0045.UK (H). M. Blackburn was supported by the UK Natural Environment Research Council.

Topical Editor J.-P. Duvel thanks W. J. Gutowski and P. H. Stone for their help in evaluating this paper.

\section{Appendix}

The energetics analysis used here follows that of Pearce (1978) with modifications to remove minor inconsistencies by Blackburn (1983). The potential and kinetic energies, their sources and the conversions between them referred to in the text and depicted in the figures are defined as follows.

$$
\begin{aligned}
& K_{z}=\frac{1}{2}\left(\overline{\left[\overline{[u]^{2}}\right.}+\widetilde{[v]^{2}}\right) \quad K_{e}=\frac{1}{2}\left(\overline{\left[u^{* * 2}\right]}+\overline{\left[v^{* * 2}\right]}\right) \\
& N_{s}=c_{p} \frac{\overline{\left[\widetilde{T]^{\prime 2}}\right.}}{2 \hat{T}}, \quad N_{z}=c_{p} \frac{\overline{[]^{\prime 2}}}{2 \hat{T}}, \quad N_{e}=c_{p} \frac{\overline{\left[T^{* * 2}\right]}}{2 \hat{T}}
\end{aligned}
$$

$$
\begin{aligned}
& G_{s}=\frac{\overline{[q]^{\prime}\left[\widetilde{T]^{\prime}}\right.}}{\hat{T}}, \quad G_{z}=\frac{\overline{[q]^{\prime \prime}[]^{\prime \prime}}}{\hat{T}}, \quad G_{e}=\frac{\overline{\left[q^{* *} T^{* *}\right]}}{\hat{T}}
\end{aligned}
$$

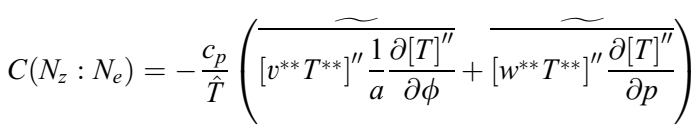

$$
\begin{aligned}
& \left.C\left(N_{e}: K_{e}\right)=-R \overline{\frac{\left[w^{* *} T^{* *}\right]}{p}}\left(1-\overline{\frac{p}{\kappa \hat{T}} \frac{\partial \widetilde{T}^{\prime}}{\partial p}}\right)\right)
\end{aligned}
$$

where:

$\hat{a}$ is the global average and $a^{*}$ its departure

$[a]$ is the zonal average and $a^{* *}$ its departure

$\tilde{a}$ is the meridional average and $a^{\prime \prime}$ its departure

$\bar{a}$ is the vertical average and $a^{\prime}$ its departure.

$q$ is the heating rate $\left(\mathrm{J} \mathrm{kg}^{-1} \mathrm{~s}^{-1}\right)$

$\kappa=R / c_{p}$

Values are given in the text and the figures in units of $10^{5} \mathrm{~J} \mathrm{~m}^{-2}$ for energies, and units of $10^{5} \mathrm{~J} \mathrm{~m}^{-2}$ per day for conversions. Totals for a given time period shown in Fig. 6 are the averages of these expressions with respect to time, pressure, latitude and longitude, with a cosine latitude geometrical weighting.

\section{References}

Betts, A. K., A new convective adjustment scheme I: Observational and theoretical basis, Q. J. R. Meteorol Soc., 112, 677-691, 1986.

Blackburn M., PhD Thesis, University of Reading, Earley Gate, Reading RG6 2AU, UK, 1983.

Boer, G. J., Budgets and balances in a warmer world. The seventeenth Stanstead Seminar: the role of large-scale extratropical dynamics in climate change, Lennoxville, PQ, Canada. Dept of Atmospheric and Oceanic Sciences, McGill University, Montreal, pp 1-6, 1993.

Branscome, L. E., and W. J. Gutowski, The impact of doubled $\mathrm{CO}_{2}$ on the energetics and hydrologic processes of midlatitude transient eddies, Clim. Dyn., 8, 29-37, 1992.

Chang, C. B., D. J. Perkey, and C. W. Kreitzberg, A numerical case study of the effects of latent heating on a developing wave cyclone, J. Atmos. Sci., 39, 1555-1570, 1982.

Fantini, M., Moist Lady waves in a quasigeostrophic threedimensional model, J. Atmos. Sci., 52, 2473-2485, 1995.

Gutowski, W. J., Jr., L. E. Branscome, and D. A. Stewart, Life cycles of moist baroclinic eddies, J. Atmos. Sci., 49, 306-319, 1992.

Gutowski, W. J., P. C. Nendick and L. E. Branscome, Sensitivity of transient eddies to climate change in the CCC general circulation model., Atmos-Ocean, 33, 753-770, 1995.

Hall, N. M. J., B. J. Hoskins, P. J. Valdes, and C. A. Senior, Storm tracks in a high-resolution GCM with doubled carbon dioxide, Q. J. R. Meteorol. Soc., 120, 1209-1230, 1994.

Hall, N. M. J, B. Dong and P. J. Valdes, Atmospheric equilibrium, instability and energy transport at the last glacial maximum, Clim. Dyn., 12, 497-511, 1996.

Held, I. M., Large-scale dynamics and global warming, Bull. Am. Meteorol. Soc., 74, 1993, 228-241, 1993.

Hoskins, B. J., and A. J. Simmons, A multi-layer spectral model and the semi-implicit method, Q.J. R. Meteorol. Soc., 101, 637655, 1975.

James, I. N. and Gray, L. J., Concerning the effect of surface drag on the circulation of a baroclinic planetary atmosphere, $Q$. J.R. Meteorol. Soc., 112, 1231-1250, 1986.

Kalnay, E., Kanamitsu, M., Kitsler, R., Collins, W., Dearen, D., Gandu, L., Iredell, M., Saha, S., White, G., Woolen, J., Zhu, Y., Chelliah, M., Ebisuzaki, W., Higgins, W., Janowick, J., Mo, K-C., Ropelewski, C., Wang, J., Leetma, A., Reynolds, R., 
Jenne, R., and Joseph, D., The NCEP/NCAR 40-Year reanalysis project, Bull. Am. Meteorol. Soc. 77, 437-471, 1996.

Mitchell, J. F. B., C. A. Senior and W. J. Ingrams, $\mathrm{CO}_{2}$ and climate: a missing feedback ?, Nature, 341, 132-134, 1989.

Mitchell, J. F. B., S. Manabe, T. Tokioka and V. Meleshko, Equilibrium climate change, in Climate change-the IPCC scientific assessment, Eds. J. T. Houghton, G. J. Jenkins and J. J. Ephraums. Cambridge University Press, Cambridge, 1990.

Pavan, V., Sensitivity of a multi-layer quasi-geostrophic $\beta$-channel to the vertical structure of the equilibrium meridional temperature gradient, Q. J. R. Meteorol. Soc., 122, 55-72, 1995.

Pearce, R. P., On the concept of available potential energy, Q. J. R. Meteorol. Soc., 104, 737-755, 1978.
Shine, K.P., and A. Sinha, Sensitivity of the earth's climate to height dependent changes in the water vapour mixing ratio, Nature, 354, 382-384, 1991.

Simmons, A. J., and B. J. Hoskins, The life cycles of some nonlinear baroclinic waves, J. Atmos. Sci., 35, 414-432, 1978.

Stephenson, D. B., The impact of changing the horizontal diffusion scheme on the climatology of a perpetual January general circulation model, Q. J. R. Meteorol. Soc., 121, 211-226, 1995.

Thorncroft, C. D., B. J. Hoskins and M. E. McIntyre, Two paradigms of baroclinic wave life-cycle behaviour, Q. J.R. Meteorol. Soc., 119, 17-55, 1993. 